\title{
Robust tracking control design for fluid traffic dynamics
}

\author{
Liudmila Tumash, Carlos Canudas-de-Wit and Maria Laura Delle Monache
}

\begin{abstract}
The paper is devoted to the boundary control of the traffic system described by the LWR model with a triangular fundamental diagram and a space-dependent indomain unknown disturbance, which can be described as an inhomogeneous transport equation. The controller design strategy aims first at stabilizing the deviation from the desired time-dependent trajectory and then at minimizing the deviation in the sense of two possible space-norms (i.e. $L_{2}$ and $L_{\infty}$ ). Numerical simulations for both $L_{2}$ and $L_{\infty}$ minimization cases are presented to evaluate the improvements obtained with this control design.
\end{abstract}

\section{INTRODUCTION}

One of the most famous models used to describe the evolution of traffic on a macroscopic scale is the so-called $L W R$ model. It was first introduced by Lighthill, Whitham [1] and Richards [2] in the fifties, who used fluid dynamics to model traffic flow. The LWR model is the first macroscopic model, which describes the spatio-temporal evolution of the traffic density defined along a freeway. From the mathematical viewpoint, it corresponds to a scalar hyperbolic conservation law with a concave flux function.

In the past decades, several papers appeared proposing different mathematical techniques in the control of conservation laws and hyperbolic PDEs. The most relevant techniques are backstepping [3], Lyapunov-based [3] and optimal control methods [4]. These techniques have been applied to optimize the traffic flow. One of the most relevant topics widely studied nowadays is the boundary control, e.g. ramp metering [5].

However, most of the previously cited works have addressed the homogeneous (ideal) case, where the discrepancies between model and system are often ignored. This work addresses two seldom studied issues: how to track time-space varying desired profiles, and how to handle uncertainties due to possible model mismatch. In particular, we study an optimal boundary control problem on a single road of finite length with space-dependent disturbance by tracking the desired target traffic state (vehicle density). Such a disturbance function can be interpreted as follows. Imagine a single main road that is controlled at its entrance. Then

This work was supported by the funding from the European Research Council (ERC) under the European Union's Horizon 2020 research and innovation programme (grant agreement 694209)

L. Tumash is with Univ. Grenoble Alpes, CNRS, Inria, Grenoble INP, GIPSA-lab, 38000 Grenoble, France, liudmila.tumash@gipsa-lab.fr

C. Canudas-de-Wit is with Univ. Grenoble Alpes, CNRS, Inria, Grenoble INP, GIPSA-lab, 38000 Grenoble, France, carlos.canudas-de-wit@gipsa-lab.grenoble-inp.fr

M. L. Delle Monache is with Univ. Grenoble Alpes, Inria, CNRS, Grenoble INP, GIPSA-Lab, 38000 Grenoble, France ml.dellemonache@inria.fr the disturbance corresponds to the unknown change in the number of vehicles coming from minor in- and out-coming side roads, which is definitely a realistic situation in traffic.

Some of the studies related to the disturbance rejection problem in this context have been devoted to effecting the disturbance on one boundary from the other [6], [7], [8]. For example, in [7] they use the sliding mode control in order to stabilize the hyperbolic system with boundary input disturbance. In [6] they designed a controller which is able to reject the disturbance only at the boundary where the disturbance acts. Later in [8] they generalized the results by deriving a controller for the disturbance rejection at an arbitrary point in the domain. A model reference adaptive control problem has been solved for hyperbolic PDEs in [9]. Therein, the authors considered harmonic disturbances with known frequencies and designed a filter-based control law. In a related work on robust control design of the systems of conservation laws [10] they state the problem of stabilization of a steady state profile. Boundary control has been addressed previously for example, in [11] where the density is driven to an constant equilibrium.

The main contribution of this paper is the optimal boundary controller which leads to the attenuation of the general in-domain space-dependent disturbance. To the best of our knowledge, this is the first result devoted to a robust controller tracking a space- and time-dependent desired traffic density. The control design is based on two components. These are feedforward control used for tracking the trajectory and an optimal feedback component used to optimize the asymptotic spatial $L_{2}$ and $L_{\infty}$ norms of the deviation from the desired trajectory. We show that the optimal feedback term takes different forms according to the norm to be minimized. The feedback law is given in its implicit (but computationally feasible) form which is independent of the knowledge of the disturbance. In addition, we also compute the $L_{2}$ and $L_{\infty}$ gains resulting from the application of the respective control laws. These gains are particular, since they do not depend on the controller gains but on the systems physical parameters (length of the road stretch and the parameters of the fundamental diagram).

This paper is organized as follows. We start by defining the LWR model with triangular flux function in Section II and state the disturbance rejection problem. Moreover, we discuss the solution of the error system and analyse its steady state. We state the main results in Section IV and V. In Section VI we provide a numerical example that verifies the theoretical results. The concluding remarks are given in Section VII. 


\section{Preliminaries AND PRoblem Statement}

We first fix the notations used in the paper. We introduce the following norms with respect to the space variable $x$ as time $t \rightarrow \infty$. For a function $f(x, t) \in[0, L] \times \mathbb{R}_{+}$the $L_{2}$ and $L_{\infty}$ norms are defined as

$$
\begin{aligned}
\|f(\cdot, t)\|_{2} & :={\sqrt{\int_{0}^{L} f^{2}(x, t) d x}} & \forall t \in \mathbb{R}_{+}, \\
\|f(\cdot, t)\|_{\infty} & :=\sup _{x \in[0, L]}|f(x, t)| & \forall t \in \mathbb{R}_{+},
\end{aligned}
$$

where sup (inf) indicate the essential supremum (infinum).

The LWR model is based on the conservation of the number of vehicles and can be expressed as

$$
\frac{\partial \rho(x, t)}{\partial t}+\frac{\partial \Phi(\rho(x, t))}{\partial x}=0, \quad \forall(x, t) \in[0, L] \times \mathbb{R}_{+}
$$

where $\rho(x, t)$ indicates the traffic density and $\Phi(\rho):=\rho v(\rho)$ is the flux function. Typical flux functions are obtained from an empirical relationship between the traffic density and the flow known as the fundamental diagram (FD), see a detailed overview in [12]. We assume that $\Phi(\rho):\left[0, \rho_{\max }\right] \rightarrow \mathbb{R}$ is a Lipschitz continuous and concave function with a unique maximum $\Phi_{\max }$ (capacity) attained at $\rho_{c}$ (critical density).

In this article, we use a triangular (bilinear) fundamental diagram, see Figure 1, proposed by Daganzo [13]:

$$
\Phi(\rho)= \begin{cases}v \rho, & \rho \in \Omega_{f} \\ -w\left(\rho-\rho_{\max }\right), & \rho \in \Omega_{c}\end{cases}
$$

where $\Omega_{f}:=\left[0, \rho_{c}\right]$ is the free-flow regime and $\left.\left.\Omega_{c}:=\right] \rho_{c}, \rho_{\text {max }}\right]$ the congested regime. In (2) the critical density is defined as $\rho_{c}=\frac{w}{v+w} \rho_{\max }$, where $v$ and $w$ are velocities of a kinematic wave propagating forwards and backwards, respectively.

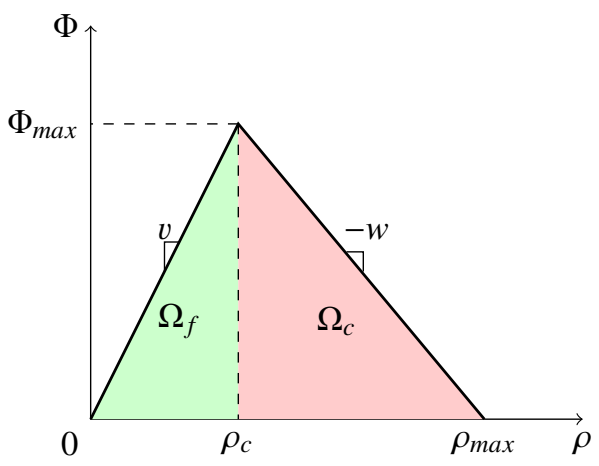

Fig. 1: Triangular Fundamental Diagram, in green the free flow regime $\Omega_{f}$ and in red the congested regime $\Omega_{c}$

The goal of this paper is to design a robust boundary control law $u(t)$ for equation (1) with disturbance such that we can drive the system to a desired time-dependent state. Let us now describe the problem in mathematical terms.

We assume that the density can be only in one regime at a time, and for simplicity let us introduce a generalized notation for the current regime

$$
r:= \begin{cases}f, & \text { if } \rho \in \Omega_{f}, \\ c, & \text { if } \rho \in \Omega_{c} .\end{cases}
$$

Then we can define the free flow regime

$$
\Omega_{r}:=\Omega_{f}, \quad x_{r}:=0, \quad v_{r}:=v .
$$

Similarly, the congested regime is denoted as

$$
\Omega_{r}:=\Omega_{c}, \quad x_{r}:=L, \quad v_{r}:=-w,
$$

where $x_{r}$ indicates the boundary where the control will be implemented and $v_{r}$ the wave's speed in the chosen regime.

\section{A. Inhomogeneous transport equation with boundary control}

First, we introduce a disturbance $\delta(x):[0, L] \rightarrow \mathbb{R}$ into (1). We assume that the disturbance is unknown and bounded. The initial-boundary value problem (IBVP) reads:

$$
\Sigma_{\rho}=\left\{\begin{array}{l}
\frac{\partial \rho(x, t)}{\partial t}+v_{r} \frac{\partial \rho(x, t)}{\partial x}=\delta(x), \\
\rho(x, 0)=\rho_{0}(x), \\
\rho\left(x_{r}, t\right)=u(t),
\end{array}\right.
$$

where $u(t)$ is the control action applied at the boundary. Note that we control the upstream $x_{r}=0$ boundary if the kinematic wave propagates forwards (free-flow regime) or at the downstream $x_{r}=L$ boundary if the wave moves backwards (congested regime). Physically, $\delta(x)$ corresponds to the additional unknown vehicle density per time unit.

\section{B. Admissible equilibria}

Let us now define the desired density $\rho_{d}(x, t)$ that we want to reach via the boundary control as a time-dependent equilibrium (desired trajectory). For $\rho_{d}(x, t)$ to be admissible, it must be a solution of $\Sigma_{\rho_{d}}$, which the same IBVP as (3) but a homogeneous one $(\delta(x)=0)$. Moreover, $\rho_{d}(x, t)$ must remain in the prescribed regime $\forall(x, t), \rho_{d}(x, t):[0, L] \times \mathbb{R}_{+} \rightarrow \Omega_{r}$.

\section{IBVP for the density error}

Let us introduce the density error as $\tilde{\rho}(x, t)=\rho(x, t)-$ $\rho_{d}(x, t)$. Since the problems are linear, we can subtract $\Sigma_{\rho_{d}}$ from $\Sigma_{\rho}$ and obtain the following IBVP for $\tilde{\rho}(x, t)$

$$
\Sigma_{\tilde{\rho}}=\left\{\begin{array}{l}
\frac{\partial \tilde{\rho}(x, t)}{\partial t}+v_{r} \frac{\partial \tilde{\rho}(x, t)}{\partial x}=\delta(x), \\
\tilde{\rho}(x, 0)=\tilde{\rho}_{0}(x) \\
\tilde{\rho}\left(x_{r}, t\right)=u(t)-\rho_{d_{x_{r}}}(t),
\end{array}\right.
$$

where $\rho_{d_{x_{r}}}$ is the desired state at the boundary $x_{r}$. For simplicity and without the loss of generality, in the following we show the results only for control at the upstream boundary, i.e. $x_{r}=0$ (then $v_{r}=v$ ). The results for $x_{r}=L,\left(v_{r}=-w\right.$ ) can be obtained in an equivalent way.

\section{Control design}

We aim at designing a boundary control law that can be schematically represented as in Figure 2. Thus, the input is a sum of feedforward term $u^{f f}$ and feedback term $u^{f b}$

$$
u=u^{f f}+u^{f b} .
$$

For simplicity of notations, the arguments of the controls are omitted, and in the following they will be included only if not clear from the context. 


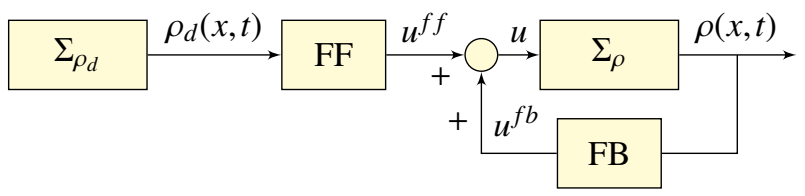

Fig. 2: Control scheme

Remark. Note that the feedforward term is designed to track the desired trajectory $\rho_{d}(x, t)$, while the feedback term is designed for disturbance attenuation.

\section{E. Solution of $\Sigma_{\tilde{\rho}}$}

Let us now consider (4). Applying the method of characteristics [14], we find that its solution $\tilde{\rho}(x, t)$ evolves along the characteristic lines as

$$
\tilde{\rho}(x, t)=\left\{\begin{array}{l}
\tilde{\rho}(x-v t, 0)+\Delta(x)-\Delta(x-v t), \quad \forall t \in\left[0, \frac{x}{v}\right) \\
\tilde{\rho}\left(0, t-\frac{x}{v}\right)+\Delta(x), \quad \forall t \in\left[\frac{x}{v},+\infty\right)
\end{array}\right.
$$

where the function $\Delta(x)$ is the integral of the disturbance:

$$
\Delta(x)=\frac{1}{v} \int_{0}^{x} \delta(s) d s,
$$

which is the disturbance accumulated along the space.

Remark. Note that $t=\frac{L}{v}$ is the minimum time for solutions at $t=0$ to propagate till the end of the $\operatorname{road} x=L$, that is why in the following we consider the solutions only for $t \geq \frac{L}{v}$.

Let us rewrite (6) using the control variables introduced in (4), i.e. using $\tilde{\rho}\left(0, t-\frac{x}{v}\right)=u\left(t-\frac{x}{v}\right)-\rho_{d_{0}}\left(t-\frac{x}{v}\right)$ :

$$
\tilde{\rho}(x, t)=u\left(t-\frac{x}{v}\right)-\rho_{d_{0}}\left(t-\frac{x}{v}\right)+\Delta(x) .
$$

Note that the only time-dependency in (8) is due to presence of the time-varying trajectory $\rho_{d_{0}}(t)$, whose effect can be compensated using the feedforward term, as we may do if $\Delta(x)=0$. Therefore, from now on we set $u^{f f}(t)=\rho_{d_{0}}(t)$, and write the solution of (8) only as a function of feedback term:

$$
\tilde{\rho}(x, t)=u^{f b}\left(t-\frac{x}{v}\right)+\Delta(x) .
$$

We also introduce the notation

$$
\tilde{\rho}_{\infty}(x)=\lim _{t \rightarrow \infty} \tilde{\rho}(x, t), \quad u_{\infty}^{f b}=\lim _{t \rightarrow \infty} \tilde{\rho}_{0}(t)
$$

Then the density error solution (9) has the following asymptotic relation for $t \rightarrow \infty$ :

$$
\tilde{\rho}_{\infty}(x)=u_{\infty}^{f b}+\Delta(x)
$$

\section{F. Problem statement}

The role of $u^{f b}$ is thus to ensure that $\tilde{\rho}(x, t) \rightarrow \tilde{\rho}_{\infty}(x)$, and $u_{\infty}^{f b}$ is such that the effect of the cumulated disturbance $\Delta(x)$ is minimized in the sense of $L_{2}$-space norm (Problem 1) and $L_{\infty}$-space norm (Problem 2). This is formalized as follows:

Problem 1,2. Find the optimal control law $u^{*}$ in the form (5), such that: i) $\tilde{\rho}(x, t) \rightarrow \tilde{\rho}_{\infty}(x)$

ii) Problem 1: $u^{*}=\operatorname{argmin}_{u \in \Omega_{r}}\left\|\tilde{\rho}_{\infty}(x)\right\|_{2}^{2}$.

Problem 2: $u^{*}=\operatorname{argmin}_{u \in \Omega_{r}}\left\|\tilde{\rho}_{\infty}(x)\right\|_{\infty}$.

Note that due to the presence of $\delta(x)$, we can not drive $\tilde{\rho}(x, t)$ to zero as $t \rightarrow \infty$ by acting only from the boundary.

\section{Control design for the Problem 1}

Let us now consider the inhomogeneous error system $\Sigma_{\tilde{\rho}}$ given by (4). We first prove that a constant $u^{f b}$ suffices for statement $i$ ), and then we derive the optimal value of $u^{f b}$ that satisfies statement ii) of Problem 1.

Lemma 1. Let $u(t)=u^{f f}(t)+u_{\infty}^{f b}$, with $u^{f f}(t)=\rho_{d_{0}}(t)$, and $u_{\infty}^{f b}$ being some constant. Then the following statement holds:

$$
\lim _{t \rightarrow \infty}\left\|\tilde{\rho}(x, t)-\tilde{\rho}_{\infty}(x)\right\|_{2}=0
$$

Proof. Similar to [15], we define the following Lyapunov function candidate

$$
V(t)=\frac{1}{2} \int_{0}^{L} e^{-v x}\left(\tilde{\rho}(x, t)-\tilde{\rho}_{\infty}(x)\right)^{2} d x,
$$

where $e^{-v x}$ is a weighting function. The time derivative is

$$
\begin{aligned}
\dot{V}(t) & =\int_{0}^{L} e^{-v x}\left(\tilde{\rho}(x, t)-\tilde{\rho}_{\infty}(x)\right) \frac{\partial \tilde{\rho}(x, t)}{\partial t} d x \\
& =\int_{0}^{L} e^{-v x}\left(\tilde{\rho}(x, t)-\tilde{\rho}_{\infty}(x)\right)\left(\delta(x)-v \frac{\partial \tilde{\rho}(x, t)}{\partial x}\right) d x .
\end{aligned}
$$

From (7), (10), and the fact that $u_{\infty}^{f b}$ is not $x$-dependent, the derivative of $\tilde{\rho}_{\infty}(x)$ with respect to $x$ is

$$
\frac{\partial \tilde{\rho}_{\infty}(x)}{\partial x}=\frac{1}{v} \delta(x),
$$

and thus we get

$$
\dot{V}(t)=-v \int_{0}^{L} e^{-v x}\left(\tilde{\rho}(x, t)-\tilde{\rho}_{\infty}(x)\right) \frac{\partial\left(\tilde{\rho}(x, t)-\tilde{\rho}_{\infty}(x)\right)}{\partial x} d x .
$$

Integration by parts yields

$$
\begin{aligned}
\dot{V}(t)= & \frac{v}{2}\left(\tilde{\rho}(0, t)-\tilde{\rho}_{\infty}(0)\right)^{2}-\frac{v e^{-v L}}{2}\left(\tilde{\rho}(L, t)-\tilde{\rho}_{\infty}(L)\right)^{2}- \\
& v^{2} V(t) \leqslant \frac{v}{2}\left(u_{\infty}^{f b}-\tilde{\rho}_{\infty}(0)\right)^{2}-v^{2} V(t) .
\end{aligned}
$$

where the last inequality comes from the fact that $u(t)=$ $\rho(0, t)=u^{f f}(t)+u_{\infty}^{f b}=\rho_{d_{0}}(t)+u_{\infty}^{f b}$, and hence $\tilde{\rho}(0, t)=u_{\infty}^{f b}$. It follows then from (10) with $\Delta(0)=0$ that

$$
\dot{V}(t) \leq \frac{v}{2}(\Delta(0))^{2}-v^{2} V(t)=-v^{2} V(t) .
$$

Lemma statement follows directly from $\left\|\tilde{\rho}(x, t)-\tilde{\rho}_{\infty}(x)\right\|_{2}^{2} \leqslant$ $2 V(t) e^{v L}$, thus $V(t) \rightarrow 0$ implies $\left\|\tilde{\rho}(x, t)-\tilde{\rho}_{\infty}(x)\right\|_{2} \rightarrow 0$.

The following theorem completes the previous result and gives the optimal form of $u^{f b}$. The final control is presented in a computable form, as the optimal value of $u^{f b}$ depends on the unmeasured disturbance. 
Theorem 1. For the density error $\tilde{\rho}(x, t)$ given by IBVP (4) the optimal boundary controller minimizing the limit of its $L_{2}$ norm as $t \rightarrow \infty$ is given by

$$
\begin{gathered}
u^{*}=u^{f f}+\bar{u}^{f b}, \quad \text { where } u^{f f}=\rho_{d_{0}}(t) \text { and } \\
\bar{u}^{f b}=\left\{\begin{array}{lrr}
0 & \text { if } & 0 \leq t<L / v \\
-\frac{1}{L} \int_{0}^{L}\left(\rho(x, t)-u^{*}\left(t-\frac{x}{v}\right)\right) d x & \text { if } & t \geq L / v
\end{array}\right.
\end{gathered}
$$

Proof. First, note that the minimization over $u^{*}$ in statement ii) in Problem 1 is equivalent to the minimization over $u^{f b}=$ $u_{\infty}^{f b}=$ constant, with $u^{*}=u^{f f}(t)+\bar{u}^{f b}$, i.e.

$$
\bar{u}^{f b}=\underset{u \in \Omega_{r}}{\operatorname{argmin}}\left\|u^{f b}+\Delta(x)\right\|_{2}^{2}=\underset{u \in \Omega_{r}}{\operatorname{argmin}} \int_{0}^{L}\left(u^{f b}+\Delta(x)\right)^{2} d x .
$$

where we have used relation (10).

Expanding the quadratic form in the integral, we obtain

$$
\left(u^{f b}\right)^{2} L+2 u^{f b} \int_{0}^{L} \Delta(x) d x+\int_{0}^{L} \Delta^{2}(x) d x .
$$

At this point it is easy to compute $\bar{u}^{f b}$ minimizing the quadratic form (15), whereby we obtain an optimal value:

$$
\bar{u}^{f b}=-\frac{1}{L} \int_{0}^{L} \Delta(x) d x .
$$

This expression corresponds to the subtraction of the mean value of the cumulative disturbance. However, we should recall that the function $\Delta(x)$ is unknown. However, for $t \rightarrow$ $\infty$ using the solution of $\tilde{\rho}(x, t)$ obtained by the method of characteristics (6), we can write

$$
\Delta(x)=\rho(x, t)-\rho_{d}(x, t)-u^{*}\left(t-\frac{x}{v}\right)+\rho_{d_{0}}\left(t-\frac{x}{v}\right) .
$$

Since the desired density $\rho_{d}(x, t)$ satisfies the homogeneous transport equation, we can again apply the method of characteristics and set $\rho_{d}(x, t)=\rho_{d_{0}}\left(t-\frac{x}{v}\right)$ :

$$
\Delta(x)=\rho(x, t)-u^{*}\left(t-\frac{x}{v}\right),
$$

which can be computed provided we know the current density $\rho(x, t)$ and the control action on previous time steps $u^{*}\left(t-\frac{x}{v}\right)$. The former can be measured instantaneously, while the latter can be stored in the memory.

The combination of (16) and (17) yields the final expression (14) stated in the theorem. This controller should be seen as a compensator of the averaged effect of disturbance on the vehicle number in the whole domain $[0, L] \times \mathbb{R}^{+}$.

Corollary 1. Controller given by (14) provides the following bound

$$
\left\|\tilde{\rho}_{\infty}(x)\right\|_{2}^{2} \leq k\|\delta(x)\|_{2}^{2}, \quad \text { with } \quad k=\frac{L^{2}}{2 v^{2}} .
$$

Proof. Substitution of (16) into (15) gives the minimum of $L_{2}$ norm of $\tilde{\rho}_{\infty}(x)$ :

$$
\left\|\bar{u}^{f b}+\Delta(x)\right\|_{2}^{2}=\int_{0}^{L} \Delta^{2}(x) d x-\frac{1}{L}\left(\int_{0}^{L} \Delta(x) d x\right)^{2} .
$$

Using (7) one obtains

$$
\left\|\bar{u}^{f b}+\Delta(x)\right\|_{2}^{2} \leq \int_{0}^{L} \Delta^{2}(x) d x=\frac{1}{v^{2}} \int_{0}^{L}\left(\int_{0}^{x} \delta(s) d s\right)^{2} d x .
$$

By Cauchy-Schwartz inequality

$\left\|\bar{u}^{f b}+\Delta(x)\right\|_{2}^{2} \leq \frac{1}{v^{2}} \int_{0}^{L} x \int_{0}^{x} \delta^{2}(s) d s d x \leq \frac{1}{v^{2}}\|\delta(x)\|_{2}^{2} \int_{0}^{L} x d x$,

and finally

$$
\left\|\bar{u}^{f b}+\Delta(x)\right\|_{2}^{2} \leq \frac{L^{2}}{2 v^{2}}\|\delta(x)\|_{2}^{2},
$$

which concludes the proof.

\section{Control Design for the Problem 2}

Theorem 2. For the density error $\tilde{\rho}(x, t)$ given by IBVP (4) the optimal boundary controller minimizing the limit of its $L_{\infty}$ norm as $t \rightarrow \infty$ is given by

$$
\begin{gathered}
u^{*}=u^{f f}+\bar{u}^{f b}, \quad \text { where } u^{f f}=\rho_{d_{0}}(t) \quad \text { and } \\
\bar{u}^{f b}= \begin{cases}0, & \text { if } 0 \leq t<\frac{L}{v}, \\
-\sup _{x}\left(\rho(x, t)-u^{*}\left(t-\frac{x}{v}\right)\right)+\inf _{x}\left(\rho(x, t)-u^{*}\left(t-\frac{x}{v}\right)\right) & \text { if } t \geqslant \frac{L}{v},\end{cases}
\end{gathered}
$$

Proof. Following the proof of Theorem 1, the minimization over $u^{*}$ in statement $i$ ) of Problem 2 is equivalent to the minimization over $u^{f b}=u_{\infty}^{f b}=$ constant:

$$
\bar{u}^{f b}=\underset{u \in \Omega_{r}}{\operatorname{argmin}}\left\|u^{f b}+\Delta(x)\right\|_{\infty}=\underset{u \in \Omega_{r}}{\operatorname{argmin}} \sup _{x \in[0, L]}\left|u^{f b}+\Delta(x)\right| .
$$

Expanding $\sup _{x \in[0, L]}\left|u^{f b}+\Delta(x)\right|$ we get

$$
\begin{aligned}
& \max \left(\sup _{x \in[0, L]}\left(u^{f b}+\Delta(x)\right),-\inf _{x \in[0, L]}\left(u^{f b}+\Delta(x)\right)\right) \\
& =\max \left(u^{f b}+\sup _{x \in[0, L]}(\Delta(x)),-u^{f b}-\inf _{x \in[0, L]}(\Delta(x))\right) .
\end{aligned}
$$

The first argument is a monotonically increasing function with respect to $u^{f b}$, while the second argument is a monotonically decreasing one. Thus, the minimum can be achieved only at the intersection point of both functions, i.e.,

$$
\bar{u}^{f b}=\frac{-\sup _{x} \Delta(x)-\inf _{x} \Delta(x)}{2}
$$

Substituting (17) in (21), we obtain the control law (19) stated in the theorem.

Corollary 2. The control law given by (19) provides the following bound

$$
\left\|\tilde{\rho}_{\infty}(x)\right\|_{\infty} \leq \mu\|\delta(x)\|_{\infty}, \text { with } \quad \mu=\frac{L}{v} .
$$

Proof. In order to estimate the bounds on $\left\|\tilde{\rho}_{\infty}(x)\right\|_{\infty}$, we need to find bounds on $\sup _{x \in[0, L]} \Delta(x)$ and $-\inf _{x \in[0, L]} \Delta(x)$. Let us start with the supremum:

$$
\begin{gathered}
\sup _{x \in[0, L]} \Delta(x)=\sup _{x \in[0, L]} \frac{1}{v} \int_{0}^{x} \delta(s) d s \leq \sup _{x \in[0, L]}\left(\frac{x}{v} \sup _{s \in[0, x]} \delta(s)\right) \leq \\
\sup _{x \in[0, L]}\left(\frac{x}{v} \sup _{s \in[0, L]} \delta(s)\right) \leq \begin{cases}0, & \text { if } \sup _{x \in[0, L]} \delta(x) \leqslant 0, \\
\frac{L}{v} \sup _{x \in[0, L]} \delta(x), & \text { if } \sup _{x \in[0, L]} \delta(x)>0 .\end{cases}
\end{gathered}
$$


For the infinum we proceed in the same way. From the bounds on supremum and infinum we distinguish three possible cases:

1) Both $\sup \delta(x)$ and inf $\delta(x)$ are positive. Then $\|\delta(x)\|_{\infty}=$ $\sup |\delta(x)|=\sup \delta(x)$. Moreover, $\left\|\tilde{\rho}_{\infty}(x)\right\|_{\infty}=$

$$
\frac{\sup \Delta(x)-\inf \Delta(x)}{2} \leq \frac{(L / v) \sup \delta(x)-0}{2}=\frac{L}{2 v}\|\delta(x)\|_{\infty} .
$$

2) Both $\sup \delta(x)$ and inf $\delta(x)$ are negative. Then $\|\delta(x)\|_{\infty}=$ $\sup |\delta(x)|=-\inf \delta(x)$. Moreover, $\left\|\tilde{\rho}_{\infty}(x)\right\|_{\infty}=$

$$
\frac{\sup \Delta(x)-\inf \Delta(x)}{2} \leqslant \frac{0-(L / v) \inf \delta(x)}{2}=\frac{L}{2 v}\|\delta(x)\|_{\infty} .
$$

3) The signs of $\sup \delta(x)$ and $\inf \delta(x)$ are different. Then $\sup \delta(x)-\inf \delta(x) \leqslant 2 \sup |\delta(x)|=2\|\delta(x)\|_{\infty}$. Then,

$$
\begin{aligned}
\left\|\tilde{\rho}_{\infty}(x)\right\|_{\infty} & =\frac{\sup \Delta(x)-\inf \Delta(x)}{2} \leqslant \frac{L}{v} \frac{\sup \delta(x)-\inf \delta(x)}{2} \\
& \leqslant \frac{L}{v}\|\delta(x)\|_{\infty} .
\end{aligned}
$$

It follows that $\left\|\tilde{\rho}_{\infty}(x)\right\|_{\infty} \leqslant \frac{L}{v}\|\delta(x)\|_{\infty}$.

\section{Numerical Simulation}

\section{A. Numerical scheme}

The analytical results are verified by the following numerical example, which shows how the feedback term $\bar{u}^{f b}$ performs if we minimize the $L_{2}$ and $L_{\infty}$ norms of $\tilde{\rho}(x, t)$ as $t \rightarrow \infty$. For simulation we use the Godunov scheme [16], which is a first order numerical method based on approximation of the density by a piecewise constant function.

We set a space interval $[0, L]$ divided into $n$ cells of size $\Delta x=L / n$, and a time step $\Delta t$. The mesh sizes $\Delta x$ and $\Delta t$ are chosen so that they satisfy the CFL condition [17]. The discrete density is then $\rho_{i}(k)$, where $i \in[1, \ldots, n]$ is the cell index and $k \in \mathbb{Z}^{+}$time index. In the numerical scheme we add the source term for $i \in\{1 . . n\}$ :

$$
\left\{\begin{array}{l}
\rho_{i}(k+1)=\rho_{i}(k)+\frac{\Delta t}{\Delta x}\left(\varphi_{i}(k)-\varphi_{i+1}(k)\right)+\Delta t \delta_{i}, \\
\rho_{1}(k+1)=\rho_{1}(k)+\frac{\Delta t}{\Delta x}\left(\varphi_{\text {in }}(k)-\varphi_{2}(k)\right)+\Delta t \delta_{1}, \\
\rho_{n}(k+1)=\rho_{n}(k)+\frac{\Delta t}{\Delta x}\left(\varphi_{n}(k)-\varphi_{\text {out }}(k)\right)+\Delta t \delta_{n},
\end{array}\right.
$$

where $\varphi_{i}(k)$ is the numerical flux defined as

$$
\varphi_{i}(k)=\min \left\{D\left(\rho_{i-1}(k)\right), S\left(\rho_{i}(k)\right)\right\},
$$

with $D\left(\rho_{i-1}(k)\right)$ and $S\left(\rho_{i-1}(k)\right)$ the demand and the supply functions, respectively, given by

$$
\begin{aligned}
& D\left(\rho_{i-1}(k)\right)=\min \left\{v \rho_{i-1}(k), v \rho_{c}\right\}, \\
& S\left(\rho_{i}(k)\right)=\min \left\{w\left(\rho_{\max }-\rho_{i}(k)\right), v \rho_{c}\right\} .
\end{aligned}
$$

Notice that we simulate the system only in one regime, thus in (23) the minimum is always resolved to the benefit of the demand function $D\left(\rho_{i-1}(k)\right)$ in case of free flow and the supply function $S\left(\rho_{i}(k)\right)$ in case of congestion.

The boundary flows $\varphi_{\text {in }}(k)$ and $\varphi_{\text {out }}(k)$ are determined by specifying the density on the cells with indices $i=0$ and $i=$ $n+1$. These are called ghost cells since they do not belong to the domain but are used to denote the state at the boundaries:

$$
\begin{aligned}
& \varphi_{\text {in }}(k)=\min \left\{D\left(\rho_{0}(k)\right), S\left(\rho_{1}(k)\right)\right\}, \\
& \varphi_{\text {out }}(k)=\min \left\{D\left(\rho_{n}(k)\right), S\left(\rho_{n+1}(k)\right)\right\} .
\end{aligned}
$$

In the uncontrolled case, we set $\rho_{0}(k)=\rho_{1}(k)$ and $\rho_{n+1}(k)=$ $\rho_{n}(k)$, which gives $\varphi_{\text {in }}(k)=\Phi\left(\rho_{1}(k)\right)$ and $\varphi_{\text {out }}(k)=\Phi\left(\rho_{n}(k)\right)$, thus the system evolves freely. In the controlled case, we set $\rho_{0}(k)=u(k)$ in free-flow regime and $\rho_{n+1}(k)=u(k)$ in the congested one.

The simulations illustrated in subsection V-B are obtained with the following parameters setting:

$$
n=500, v=2, w=1 \rho_{\max }=1, \rho_{c}=0.33, L=1 .
$$

We fix the initial condition

$$
\rho_{0}(x)=0.5-0.15 \cos (20 \pi x),
$$

the desired state

$$
\rho_{d}(x, t)=0.55+0.15 \sin \left(\pi\left(t-\frac{L-x}{w}\right)\right)
$$

and the disturbance

$$
\delta(x)= \begin{cases}-0.02 & \text { if } x \in\left[0, \frac{L}{2}\right] \\ 0.1 & \text { otherwise. }\end{cases}
$$

Thus, we chose a system in the congested regime. Numerically, this means that $\varphi_{i}(k)=S\left(\rho_{i}(k)\right) \forall i \in[1, \ldots, n], \varphi_{\text {in }}(k)=$ $S\left(\rho_{1}(k)\right)$ since $\rho_{0}(k)=\rho_{1}(k)$ and to implement the control we set $\rho_{n+1}(k)=u(k)$, so that $\varphi_{\text {out }}(k)=S(u(k))$.

There are three possible control strategies which can be applied on the boundary of the system:

1) No control action is performed.

2) Feedforward control $u(k)=u^{f f}$ is applied.

3) Control $u(k)=u^{f f}+\bar{u}^{f b}$ is applied. For the feedback in case of (14) the integral is computed by the Riemann summation over all cells inside the domain.

\section{B. Results}

In Figure 3 we demonstrate the effect of the feedback term on disturbance attenuation acting from the downstream boundary. The uncontrolled state $\rho(x, t)$ with disturbance and the desired state $\rho_{d}(x, t)$ are shown in Figure $3 \mathrm{a}$ and $3 \mathrm{~b}$, respectively. Figure $3 \mathrm{c}$ shows $\rho(x, t)$ with the feedforward term only $\left(u=u^{f f}\right)$ obtained by setting $\rho_{d}(L, t)$ on the boundary. Finally, the state under the optimal control law (14) minimizing $\left\|\tilde{\rho}_{\infty}(x)\right\|_{2}$ is shown in Figure $3 \mathrm{~d}$. The feedback term started acting at the minimal controllability time $T=\frac{L}{w}$. Comparing Figure $3 \mathrm{c}$ and Figure $3 \mathrm{~d}$ we see that the optimally controlled density has a profile which is more similar to the desired one. In Figure 4 we can see that the optimal control law applied to minimize $\left\|\tilde{\rho}_{\infty}(x)\right\|_{\infty}$ (Figure $4 \mathrm{~b}$ ) and $\left\|\tilde{\rho}_{\infty}(x)\right\|_{2}$, (Figure 4a) performs better in both cases with respect to the case with no feedback. We also see that in both cases the norm of the density error reaches its optimal constant value for $t=2$ (which is the double value of the minimal controllability time). 


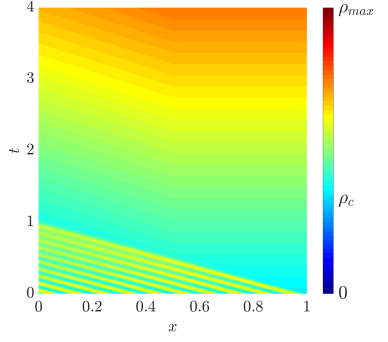

(a) State without control

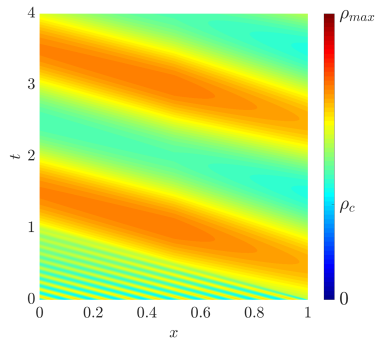

(c) Density state obtained by applying only the feedforward term of the control, $u=u^{f f}$.

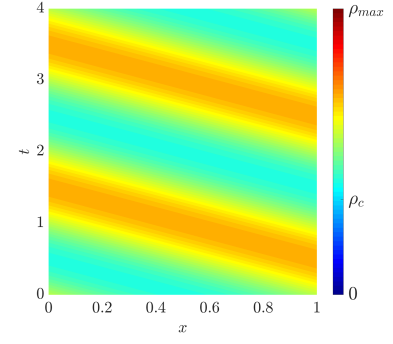

(b) Desired state given by $\Sigma_{\rho_{d}}$.

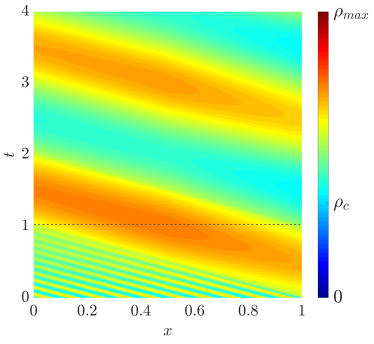

(d) Density state obtained by applying $u^{*}=u^{f f}+\bar{u}^{f b}$. The black dashed line indicates the time of adding feedback term
Fig. 3: Densitv evolution for four different choices of $u^{*}$

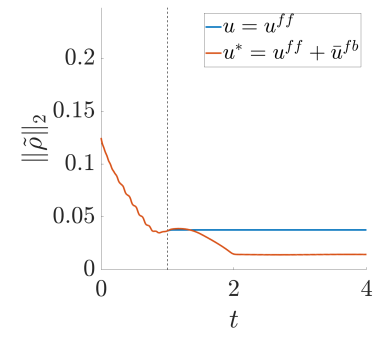

(a) $L_{2}$ norm

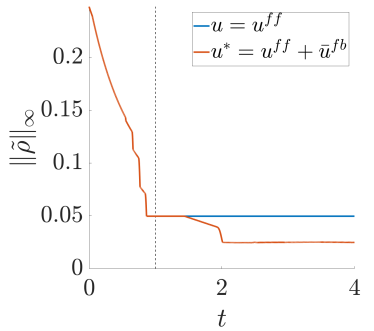

(b) $L_{\infty}$ norm
Fig. 4: The comparison of control without (blue line) and with $\bar{u}^{f b}$ (red line) for the $L_{2}$ norm and $L_{\infty}$ norm

\section{CONCLUSIONS}

We have designed a feedback control law that minimizes the deviation of the state from the desired time-dependent trajectory. The state physically corresponds to the vehicle density, which is the solution to LWR with in-domain disturbance being restricted either to the free-flow or to the congested regime. The desired trajectory solves the ideal linear LWR in the same regime. The problem was posed and solved as the disturbance attenuation problem. We obtained an optimal boundary feedback control law for the minimization of $L_{\infty}$ and $L_{2}$ norm of the density deviation. The results were verified by a numerical example, which clearly illustrates that the feedback plays an important role in the designed controller, which performs better than the one without the feedback part. The controllers for minimization of both $L_{2}$ and $L^{\infty}$ norms are optimal. The controller for the $L_{2}$ norm should be chosen if we want to minimize the meansquare deviation from the desired state, while the controller for the $L_{\infty}$ norm should be chosen if the aim is to minimize the maximal deviation from the desired state.
As for the future studies, it might be challenging to extend our analysis to more complicated problems, e.g., one could study a control problem on a network or consider a nonlinear problem (mixed case of free flow and congestion phase).

\section{ACKNOWLEDGEMENT}

The Scale-FreeBack project has received funding from the European Research Council (ERC) under the European Union's Horizon 2020 research and innovation programme (grant agreement N 694209).

\section{REFERENCES}

[1] M. J. Lighthill and G. B. Whitham, "On Kinematic Waves. II. A Theory of Traffic Flow on Long Crowded Roads," Proceedings of the Royal Society A: Mathematical, Physical and Engineering Sciences, vol. 229, no. 1178, pp. 317-345, 1955. [Online]. Available: http://rspa.royalsocietypublishing.org/cgi/doi/10.1098/rspa.1955.0089

[2] P. I. Richards, "Shock Waves on the Highway," Operations Research, vol. 4, no. 1, pp. 42-51, 1956. [Online]. Available: http://pubsonline.informs.org/doi/abs/10.1287/opre.4.1.42

[3] R. Vazquez, J. M. Coron, M. Krstic, and G. Bastin, "Local exponential $H^{2}$ stabilization of a $2 \times 2$ quasilinear hyperbolic system using backstepping," Proceedings of the IEEE Conference on Decision and Control, vol. 1, no. 4, pp. 1329-1334, 2011.

[4] D. Jacquet, M. Krstic, and C. Canudas De Wit, "Optimal control of scalar one-dimensional conservation laws," 2006 American Control Conference, no. 2, p. 6 pp., 2006.

[5] J. D. Reilly, S. Samaranayake, M. L. Delle Monache, W. Krichene, P. Goatin, and A. M. Bayen, "Adjoint-based optimization on a network of discretized scalar conservation laws with applications to coordinated ramp metering," Journal of Optimization Theory and Applications, vol. 167, no. 2, pp. 733-760, 2015.

[6] O. M. Aamo, "Disturbance rejection in $2 \times 2$ linear hyperbolic systems," IEEE Transactions on Automatic Control, vol. 58, no. 5, pp. 1095-1106, 2013.

[7] S. Tang and M. Krstic, "Sliding mode control to the stabilization of a linear $2 \times 2$ hyperbolic system with boundary input disturbance," in Proceedings of the American Control Conference. American Automatic Control Council, 2014, pp. 1027-1032.

[8] H. Anfinsen and O. M. Aamo, "Disturbance rejection in the interior domain of linear $2 \times 2$ hyperbolic systems," IEEE Transactions on Automatic Control, vol. 60, no. 1, pp. 186-191, 2015.

[9] — "Model Reference Adaptive Control of $2 \times 2$ Coupled Linear Hyperbolic PDEs," IEEE Transactions on Automatic Control, vol. 63 , no. 8, pp. 2405-2420, 2018.

[10] C. Prieur, J. Winkin, and G. Bastin, "Robust boundary control of systems of conservation laws," Mathematics of Control, Signals, and Systems, vol. 20, no. 2, pp. 173-197, 2008.

[11] S. Blandin, X. Litrico, M. L. Delle Monache, B. Piccoli, and A. M. Bayen, "Regularity and lyapunov stabilization of weak entropy solutions to scalar conservation laws," IEEE Transactions on Automatic Control, vol. 62, no. 4, pp. 1620-1635, 2017.

[12] M. Z. F. Li, "A generic characterization of equilibrium speed-flow curves," Transportation Science, vol. 42, no. 2, pp. 220-235, 2008. [Online]. Available: https://doi.org/10.1287/trsc.1070.0201

[13] C. F. Daganzo, "The cell transmission model: A dynamic representation of highway traffic consistent with the hydrodynamic theory," Transportation Research Part B: Methodological, vol. 28, no. 4, pp. 269-287, aug 1994. [Online]. Available: http://linkinghub.elsevier.com/retrieve/pii/0191261594900027

[14] L. C. Evans, Partial differential equations, ser. Graduate Studies in Mathematics. American Mathematical Society, Providence, RI, 1998, vol. 19.

[15] H. Yu and M. Krstic, "Traffic congestion control for Aw-RascleZhang model," Automatica, vol. 100, pp. 38-51, 2019. [Online]. Available: https://doi.org/10.1016/j.automatica.2018.10.040

[16] S. K. Godunov, "A difference method for numerical calculation of discontinuous solutions of the equations of hydrodynamics," Matematicheskii Sbornik, vol. 89, no. 3, pp. 271-306, 1959.

[17] R. Courant, K. Friedrichs, and H. Lewy, "On the partial difference equations of mathematical physics," IBM journal of Research and Development, vol. 11, no. 2, pp. 215-234, 1967. 